\title{
Biodegradable drug-eluting nanofiber-enveloped implants for sustained release of high bactericidal concentrations of vancomycin and ceftazidime: in vitro and in vivo studies
}

This article was published in the following Dove Press journal:

International Journal of Nanomedicine

12 September 2014

Number of times this article has been viewed

\author{
Yung-Heng $\mathrm{Hsu}^{1,2}$ \\ Dave Wei-Chih Chen' \\ Chun-Der Tai ${ }^{3}$ \\ Ying-Chao Chou ${ }^{1,2}$ \\ Shih-Jung Liu ${ }^{2}$ \\ Steve Wen-Neng Ueng \\ Err-Cheng Chan ${ }^{4}$ \\ 'Department of Orthopedic Surgery, \\ Chang Gung Memorial Hospital, \\ Guishan Township, ${ }^{2}$ Department \\ of Mechanical Engineering, Chang \\ Gung University, Guishan Township, \\ ${ }^{3}$ Graduate Institute of Medical \\ Mechatronics, Chang Gung University, \\ Guishan Township, ${ }^{4}$ School of Medical \\ Technology, Chang Gung University, \\ Guishan Township, Taiwan
}

\begin{abstract}
We developed biodegradable drug-eluting nanofiber-enveloped implants that provided sustained release of vancomycin and ceftazidime. To prepare the biodegradable nanofibrous membranes, poly $(\mathrm{D}, \mathrm{L})$-lactide-co-glycolide and the antibiotics were first dissolved in 1,1,1,3,3,3-hexafluoro-2-propanol. They were electrospun into biodegradable drug-eluting membranes, which were then enveloped on the surface of stainless plates. An elution method and a high-performance liquid chromatography assay were employed to characterize the in vivo and in vitro release rates of the antibiotics from the nanofiber-enveloped plates. The results showed that the biodegradable nanofiber-enveloped plates released high concentrations of vancomycin and ceftazidime (well above the minimum inhibitory concentration) for more than 3 and 8 weeks in vitro and in vivo, respectively. A bacterial inhibition test was carried out to determine the relative activity of the released antibiotics. The bioactivity ranged from $25 \%$ to $100 \%$. In addition, the serum creatinine level remained within the normal range, suggesting that the high vancomycin concentration did not affect renal function. By adopting the electrospinning technique, we will be able to manufacture biodegradable drug-eluting implants for the long-term drug delivery of different antibiotics.
\end{abstract}

Keywords: biodegradable nanofiber-enveloped plates, electrospinning, antibiotics, release characteristics

\section{Introduction}

Osteomyelitis in long-bone fractures presents a variety of challenges, despite the tremendous progress that has been made in treating this disease. Many factors that account for infection occurrence and persistence have been identified and a variety of antimicrobials with different activity spectra against specific pathogens have been developed. ${ }^{1-3}$ The mainstay of osteomyelitis treatment includes radical debridement, complete removal of the implant, and delivery of an effective antimicrobial agent at a sufficiently high concentration to the infected area via antibiotic-impregnated bead chains. ${ }^{4,5}$ To provide temporary stability, external skeletal fixation is one of the current surgical treatments after complete implant removal. It allows for local damage control of fractures with osteomyelitis, because it provides stability and alignment, which help control the infection. However, the substantial morbidity associated with pin-site infection and the shortening and malalignment of fractures may complicate subsequent treatment.

The use of an internal fixator that provides good stability for the fractured bones may be an ideal alternative in these situations, as long as infection does not flare up.
Correspondence: Shih-Jung Liu Biomaterials Lab, Mechanical Engineering, Chang Gung University, 259 Wen-Hwa Ist Road, Kwei-San, Tao-Yuan 333, Taiwan Email shihjung@mail.cgu.edu.tw 
Internal fixation allows for shorter hospital stays, enables patients to resume normal activities earlier, reduces the incidence of malalignment, and even supports bone healing. To prevent and/or treat the infection, the internal implants should not only stabilize the fractures but also allow for the long-term delivery of antimicrobial agents to the infected sites. A drug-eluting implant that provides local and sustained release of antibiotics is thus highly desired.

In the study reported here, we developed biodegradable poly(D,L)-lactide-co-glycolide (PLGA)/antibiotic nanofibrous membrane-enveloped plates and screws to allow for the sustained delivery of vancomycin and ceftazidime for infection treatment. Stewart et al reported the development of titanium plates bonded with vancomycin to effectively inhibit biofilm formation and support bone healing in an infected osteotomy animal model. ${ }^{6}$ To the best of our knowledge, the development of drug-eluting nanofiber-enveloped plates has not been previously reported.

Various types of carrier materials have been used based on their ability to achieve sustained bactericidal antibiotic concentrations. ${ }^{6-8}$ PLGA is a promising biodegradable material that has been used for implantable and injectable controlled-release drug-delivery systems. ${ }^{9,10}$ The copolymer has also been approved for clinical applications because it is nontoxic, instigates a minimal inflammatory response, and is biodegradable through the hydrolysis of its ester linkage to transform biocompatible lactic and glycolic acids. ${ }^{11,12}$

The choice of antibiotics for local delivery is also important. Vancomycin and ceftazidime have been commonly formulated with bone cement for osteomyelitis treatment. ${ }^{13,14}$ "Vancomycin" is a glycopeptide antibiotic that inhibits the formation of the bacterial cell wall. It is effective against Gram-positive bacteria, especially in recalcitrant staphylococcal infections that are unresponsive to penicillin or cephalosporin antibiotics. "Ceftazidime" is a third-generation cephalosporin that is effective against many Gram-negative bacteria. The combined use of these two antibiotics provides a broad-spectrum antibiotic regimen that covers not only the bacteria identified from the preoperative culture results but also unknown bacteria that might not have been detected.

To fabricate the drug-eluting implants, the biodegradable vancomycin/ceftazidime nanofibrous membranes were electospun then enveloped onto the surface of stainless plates and screws. After electrospinning, the morphology of the nanofibers was analyzed by scanning electron microscopy (SEM). The in vitro and in vivo release characteristics of the antibiotics from the nanofiber-enveloped implants were investigated. A bacterial inhibition test was carried out to determine the relative activity of the released antibiotics. In addition, a histological examination of the tissue surrounding the plates was also completed.

\section{Materials and methods Fabrication of nanofibrous membranes}

The PLGA used was commercially available (Resomer ${ }^{\circledR}$ RG 503; Boehringer Ingelheim, Ingelheim, Germany) and had a lactide-to-glycolide ratio of 50:50 and a molecular weight of 33,000 Da. Commercial-grade vancomycin hydrochloride and ceftazidime hydrate were obtained from Sigma-Aldrich (St Louis, MO, USA). The solvent 1,1,1,3,3,3-hexafluoro-2propanol (HFIP) was also purchased from Sigma-Aldrich.

The electrospinning setup used in this study consisted of a syringe and needle ( $0.42 \mathrm{~mm}$ internal diameter), a ground electrode, an aluminum sheet, and a high-voltage supply. The needle was connected to the high-voltage supply, which generated positive DC voltages and currents up to $35 \mathrm{kV}$ and $4.16 \mathrm{~mA} / 125 \mathrm{~W}$, respectively. To prepare the membranes, PLGA, vancomycin, and ceftazidime of various polymer-toantibiotic weight ratios $(260 / 10 / 10,240 / 20 / 20,230 / 25 / 25$ in $\mathrm{mg} / \mathrm{mg} / \mathrm{mg}$, respectively, denoted Groups 1-3) were dissolved in $1 \mathrm{~mL}$ of HFIP. The solution was then delivered and electrospun by a syringe pump with a volumetric flow rate of $3.0 \mathrm{~mL} / \mathrm{h}$ to obtain a $0.110 \mathrm{~mm}$-thick membrane. The distance between the needle tip and the ground electrode was $12 \mathrm{~cm}$, and the positive voltage applied to the polymer solution was $17 \mathrm{kV}$. All electrospinning experiments were carried out at room temperature. All manufactured nanofibers were placed in a vacuum oven at $40^{\circ} \mathrm{C}$ for 72 hours to evaporate the solvents.

\section{SEM analysis}

The morphology of the electrospun nanofibers was analyzed with a S-3000N scanning electron microscope (Hitachi, Tokyo, Japan) after gold coating. The average diameter and diameter distribution were determined by analyzing SEM images using a commercial image analysis program (Optimas, v 5.22, Silver Spring, MD, USA).

\section{Standard curve}

A high-performance liquid chromatography (HPLC) assay was used to determine the drug concentration standard curve. The HPLC analyses were conducted on a Hitachi L-2200 Multisolvent Delivery System. A Symmetry C8 $3.9 \mathrm{~cm} \times 150 \mathrm{~mm}$ HPLC column (Waters Corporation, Milford, MA, USA) was used to separate the vancomycin and ceftazidime. The mobile phase used for vancomycin 
contained 0.01 M heptanesulfonic acid (Fisher Scientific UK Ltd., Loughborough, UK) and acetonitrile (Mallinckrodt Pharmaceuticals, Dublin, Ireland) $(85 / 15, \mathrm{v} / \mathrm{v})$. The absorbance was monitored at a wavelength of $280 \mathrm{~nm}$ at a flow rate of $1.4 \mathrm{~mL} / \mathrm{min}$.

The mobile phase used for ceftazidime contained methanol and $5 \mathrm{mM}$ of $\mathrm{pH} 7.5$ phosphate buffer (Sigma-Aldrich) $(10 / 90, \mathrm{v} / \mathrm{v})$. The absorbance was monitored at a wavelength of $254 \mathrm{~nm}$ at a flow rate of $0.6 \mathrm{~mL} / \mathrm{min}$.

All experiments were carried out in triplicate and the sample dilutions were performed to bring the unknown concentrations into the range of the standard curve. A calibration curve was prepared for each set of measurements (correlation coefficient $>0.99$ ). The elution product was specifically identified and quantified with high sensitivity using the HPLC system.

\section{In vitro release of antibiotics}

The release characteristics of vancomycin and ceftazidime from the nanofibrous membranes were determined by using the in vitro elution method. Samples $(1 \times 1 \mathrm{~cm})$ cut from the electrospun membranes were placed in glass test tubes (one sample per test tube, total number $=3$ ) containing $1 \mathrm{~mL}$ of phosphate buffer solution ( $0.15 \mathrm{M}, \mathrm{pH} 7.4)$ each. The glass test tubes were incubated at $37^{\circ} \mathrm{C}$ for 24 hours before the membrane eluant was collected and analyzed. Fresh phosphate buffer solution $(1 \mathrm{~mL})$ was then added for the next 24-hour period, and this procedure was repeated for 35 days. The drug concentrations in the eluants were determined from the HPLC standard curve.

The relative antibiotic activity of the eluants was determined using the disk-diffusion method in nutrient broth (beef extract, peptone; Becton, Dickinson and Company, Franklin Lakes, NJ, USA). Eight microliters of membrane eluant was pipetted onto the $6 \mathrm{~mm}$ absorption disks. The disks were placed onto nutrient agar plates that were seeded with a layer of Staphylococcus aureus (ATCC65389). The inhibition zones were measured with a micrometer after 16-18 hours of incubation at $35^{\circ} \mathrm{C}$. A $50 \mu \mathrm{g} / \mathrm{mL}$ drug solution was used as a reference. The relative activity of the released vancomycin was defined as follows: relative activity $(\%)=($ diameter of sample inhibition zone)/(diameter of reference inhibition zone) $\times 100$. The bioactivity of ceftazidime on Escherichia coli (ATCC25922) was determined using the same method.

\section{Surgical procedure and animal care}

Adult New Zealand white rabbits with a mean weight of $3.5 \pm 0.3 \mathrm{~kg}$ (Animal Health Research Institute, Panchiao,
Taiwan) were used in this study. The rabbits were housed in individual cages in a temperature- and light-controlled room and given standard rabbit chow and sterilized drinking water ad libitum. All animal procedures were institutionally approved, and animal care under the supervision of a licensed veterinarian was in accordance with the regulations of the National Institute of Health of Taiwan.

The rabbits were anesthetized by intramuscular injection of Zoletil ${ }^{\circledR} 50(25 \mathrm{mg} / \mathrm{kg}$ body weight; Virbac Laboratories, Carros, France) and 2\% Rompun ${ }^{\circledR}(0.15 \mathrm{~mL} / \mathrm{kg}$ body weight; Bayer, Leverkusen, Germany). Under sterile conditions, the mid-third of the left femur was exposed through a lateral longitudinal skin incision. A cylindrical bone defect $(0.3 \times 0.3 \times 10.0 \mathrm{~mm})$ was made at the mid-third side of the left femur and obliterated with a polymethylmethacrylate (PMMA) spacer. The wound was closed with 3-0 Vicryl sutures (Johnson \& Johnson, New Brunswick, NJ, USA). After 2 weeks, the PMMA spacer was removed. A commercially available stainless plate (straight, 6-hole, $35 \mathrm{~mm}$ Mini plate; Synthes Holding AG, Solothurn, Switzerland) and screws enveloped with the PLGA/antibiotic nanofiber membrane (Figure 1) were used to fix the bone defect and the wound was then closed.

Three rabbits were euthanized by intravascular injection of lidocaine on Days 1, 3, 7, 14, 21, 28, 42, and 56 after the implantation of the drug-eluting plates. Cylindrical specimens of tissue surrounding the plate were collected from each femoral site by using a pipe with a $1.5 \mathrm{~mm}$ radius and scissors. The specimens were collected from Sites 1 to 7 , as illustrated in Figure 2. Sites 1-5 were around the plate, while Site 6 was under it. The tissue at Site 7 was collected from the bone canal with a rongeur. All specimens were centrifuged and the plasma was collected and stored at $-80^{\circ} \mathrm{C}$ until analysis. The in vivo antibiotic concentrations of the tissue samples were determined by HPLC analysis. All samples were diluted with phosphate-buffered saline and assessed according to the assay standard curve. A calibration curve was prepared for each set of the measurements (correlation coefficient $>0.99$ ).

Blood samples for determination of serum creatinine level and antibiotic concentrations were collected from the marginal ear vein using a syringe before placement of the PMMA spacers, before the implantation of the plates/screws, and before the rabbits were sacrificed. Creatinine level was determined using a Hitachi Labospect 008, while the concentrations of vancomycin and ceftazidime were determined by HPLC analysis. The collected data were used to assess the renal function of the rabbits during the experiments. The specimens harvested from Sites 1-5 were cut into $3 \mathrm{~mm}$ sections and sent for histological examination. 

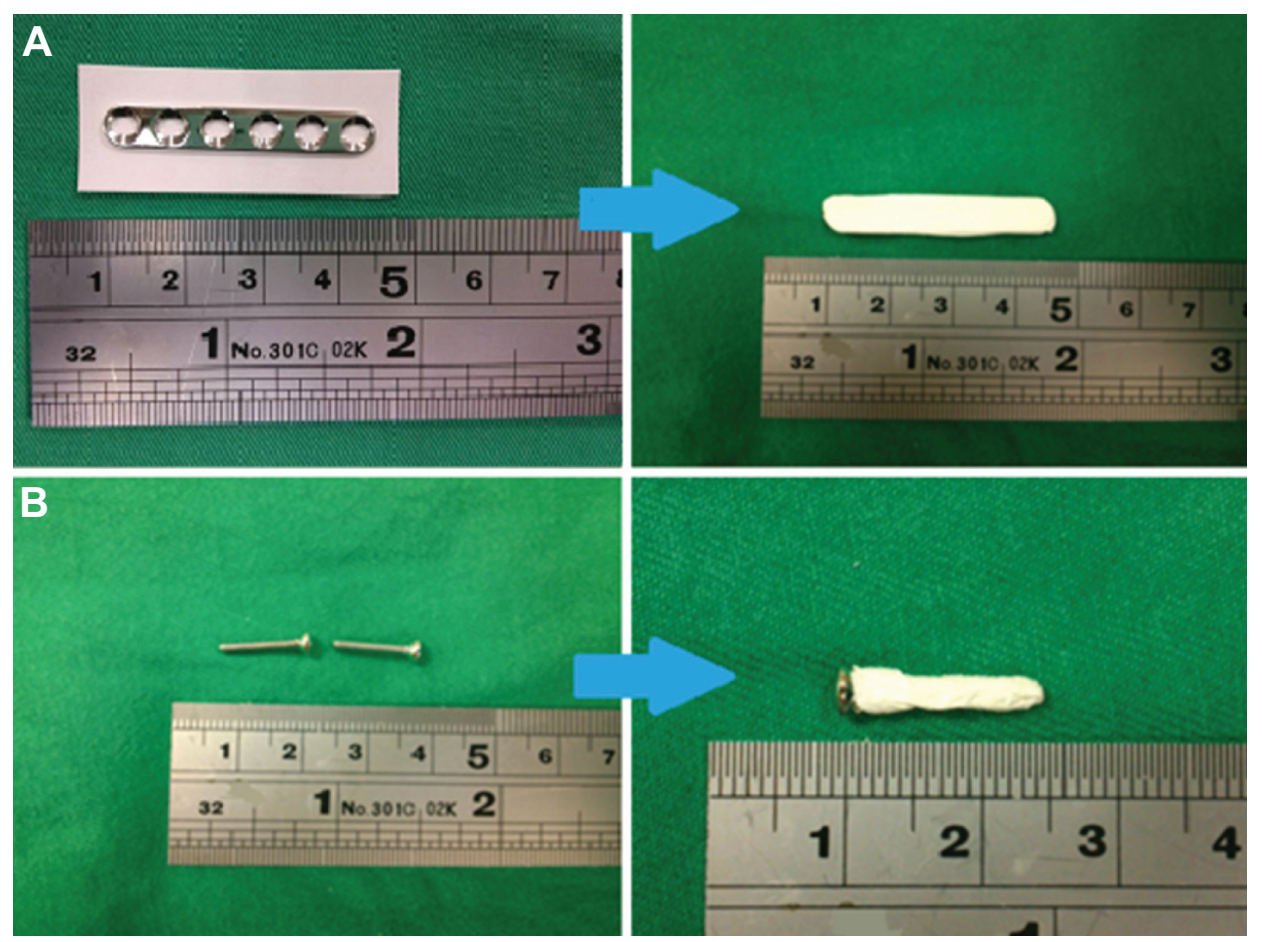

Figure I Photos of drug-eluting membrane-enveloped plate and screws.

Notes: (A) process of the plate enveloping; (B) process of the screw enveloping.

\section{Results}

Drug-eluting nanofibers were successfully fabricated by the electrospinning technique. Figure 3 shows SEM micrographs of the drug-eluting PLGA nanofibers under 20,000× magnification. The diameters of the electrospun nanofibers ranged from 80 to $630 \mathrm{~nm}$, and their porosity was high.

\section{In vitro release characteristics of the PLGA/antibiotics nanofibers}

The influence of polymer-to-antibiotic ratios on the in vitro release characteristics of the nanofibrous membranes was investigated. Three different polymer-to-antibiotic

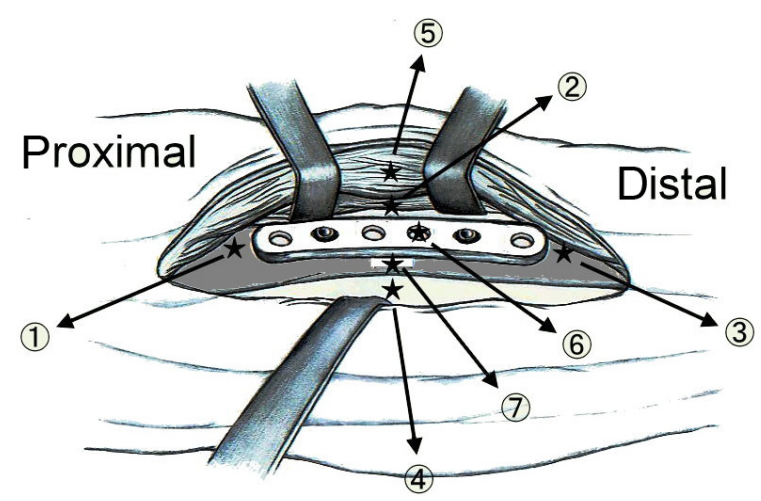

Figure 2 Schematic of the tissue-sampling sites: Site I - proximal side, Site 2 - right side, Site 3 - distal side, Site 4 - left side, Site 5 - above the implant, Site 6 - under the implant, Site 7 - inside the bone canal, Site 8 - blood. weight ratios (denoted Groups 1-3) were used in this study. The nanofibers in Group 1 were prepared using $260 \mathrm{mg}$ PLGA, $10 \mathrm{mg}$ vancomycin, and $10 \mathrm{mg}$ ceftazidime in $1 \mathrm{~mL}$ HFIP; those in Group 2 were prepared using $240 \mathrm{mg}$ PLGA, $20 \mathrm{mg}$ vancomycin, and $20 \mathrm{mg}$ ceftazidime; and those in Group 3 were prepared using $230 \mathrm{mg}$ PLGA, $25 \mathrm{mg}$ vancomycin, and $25 \mathrm{mg}$ ceftazidime. Figures 4 and 5 show the release curves of vancomycin and ceftazidime, respectively. All groups exhibited similar vancomycin-release patterns,

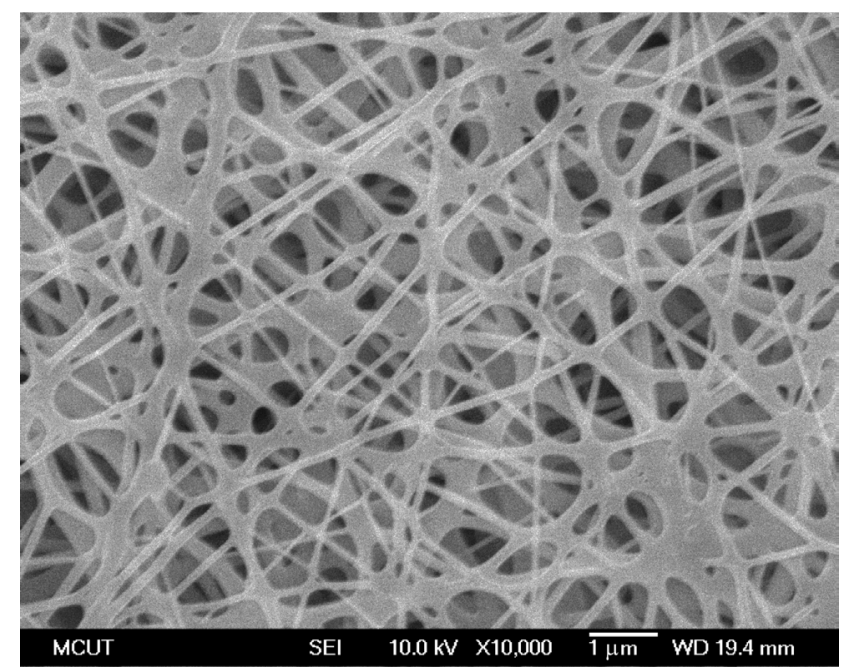

Figure 3 Morphology of the nanofibrous membrane elucidated by scanning electron microscopy. 


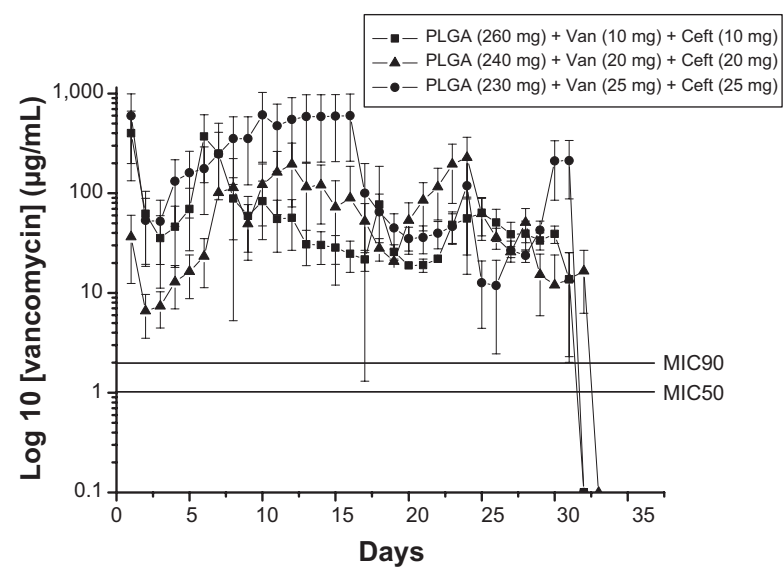

Figure 4 In vitro release characteristic of vancomycin ( Van).

Abbreviations: Ceft, ceftazidime; MIC50, minimum inhibitory concentration required to inhibit the growth of $50 \%$ of organisms; MIC 90 , minimum inhibitory concentration required to inhibit the growth of $90 \%$ of organisms; PLGA, poly(D,L)-lactideco-glycolide.

characterized by initially high release during the first 2 days, a major release peak at 10 days, and a minor release peak at 23 days, followed by more gradual and sustained drug release. In addition, all membranes exhibited the sustained release of high vancomycin concentrations (well above the minimum inhibitory concentration [MIC]) for 32 days. Group 3 had higher drug concentrations than the other two groups (Figure 4), which were mainly attributable to the higher drug loading of the membranes $(25 \mathrm{mg})$. On the other hand, the in vitro release of ceftazidime was relatively steady and only minor initial burst releases were observed. Again, the membranes in Group 3 had higher ceftazidime-release concentrations than the other groups. However, the total release period of Group 3 was 23 days, which was shorter than that of the other two groups (32 days; Figure 5). This might be because some drugs could not be completely encapsulated by the polymeric matrix as drug loading increased. Hence, the release rate of ceftazidime increased and the total release period decreased accordingly.

The bioactivities of eluted vancomycin on $S$. aureus (ATCC65389) and ceftazidime on E. coli were determined using an antibiotic disk-diffusion method. Figures $6 \mathrm{~A}$ and $\mathrm{B}$ show the bioactivities of released vancomycin and ceftazidime, respectively. The bioactivities of vancomycin and ceftazidime ranged from $25 \%$ to $100 \%$. The antibiotic bioactivity remained high after the electrospinning process. This indicates that the electrospun membrane can be an ideal drug-eluting system for the sustained release of antibiotics. Furthermore, since the Group 2 membranes of $(\mathrm{PLGA} / \mathrm{vancomycin} /$ ceftazidime $=240 / 20 / 20 \mathrm{mg})$ exhibited the most sustained release of high-concentration antibiotics, they were selected for the in vivo test.

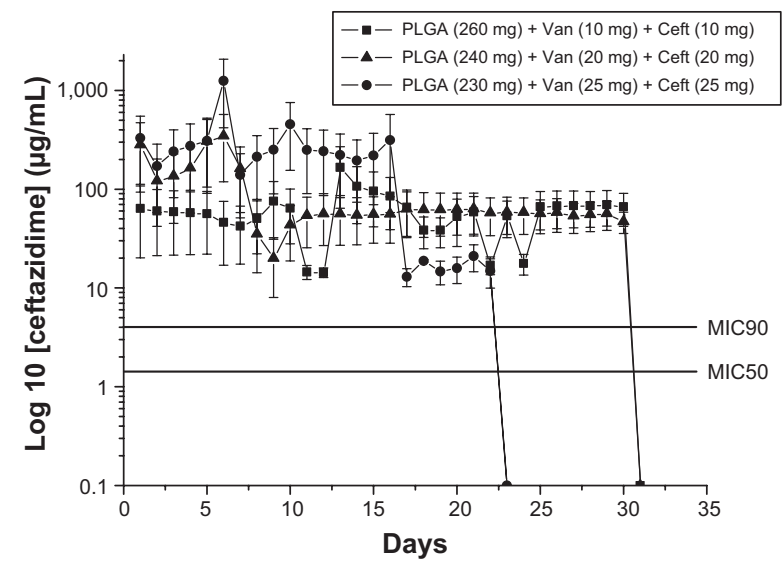

Figure 5 In vitro release characteristic of ceftazidime (Ceft).

Abbreviations: $\mathrm{Van}$, vancomycin; $\mathrm{MIC50}$, minimum inhibitory concentration required to inhibit the growth of $50 \%$ of organisms; MIC 90 , minimum inhibitory concentration required to inhibit the growth of $90 \%$ of organisms; PLGA, poly(D,L)-lactide-coglycolide.

\section{In vivo release behavior of nanofibers}

The in vivo release curves of vancomycin and ceftazidime from the membranes are shown in Figures 7 and 8, respectively. The vancomycin-release curves indicated a gradual and sustained release of the drug. In contrast, ceftazidime

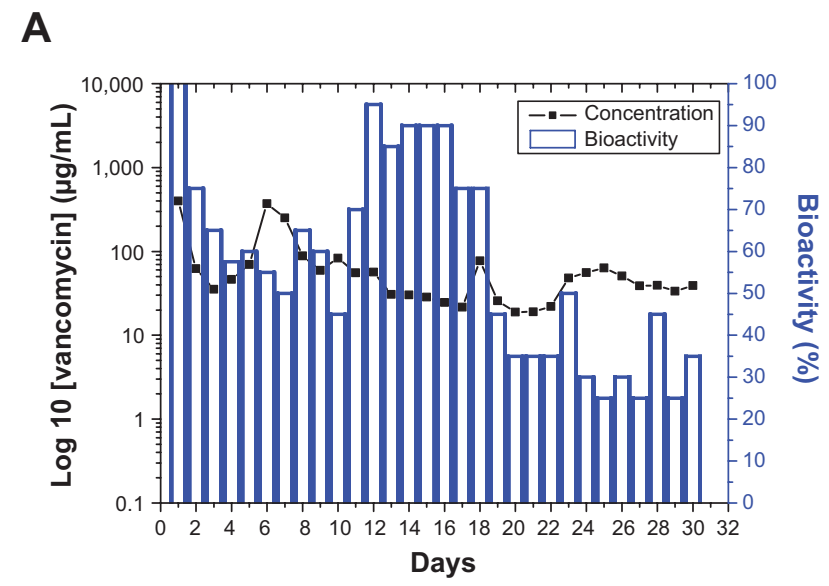

B

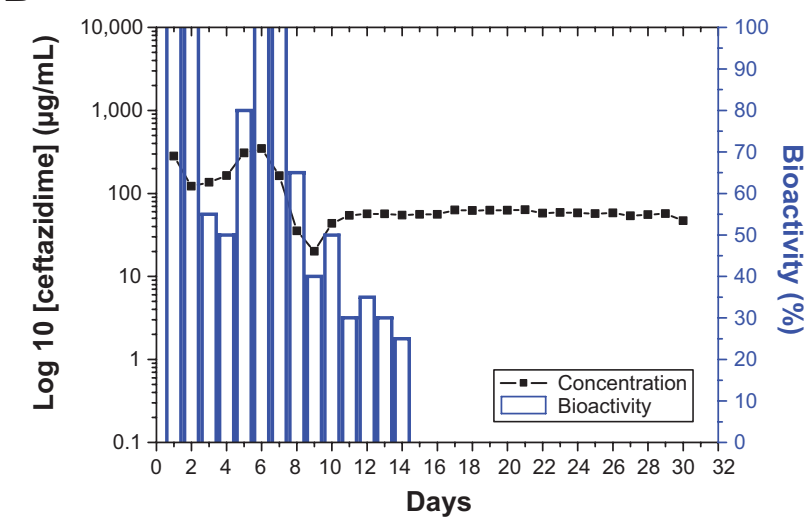

Figure 6 Bioactivity of the eluted antibiotics: (A) vancomycin, (B) ceftazidime. 


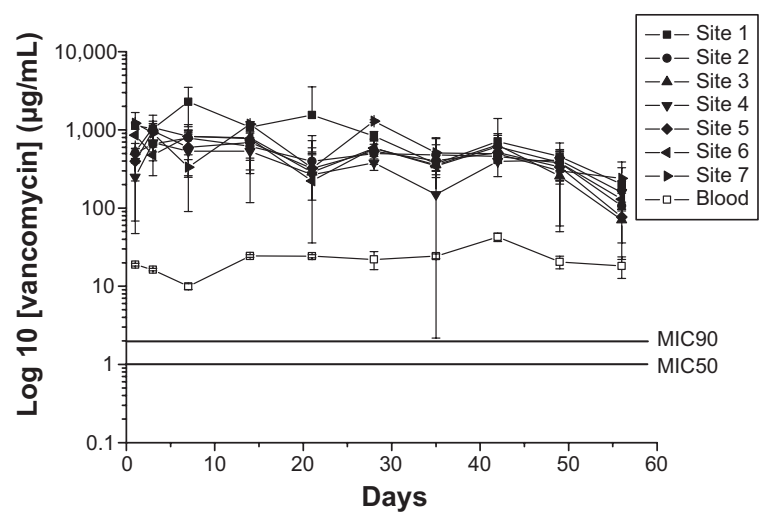

Figure 7 In vivo release behavior of vancomycin.

Abbreviations: MIC50, minimum inhibitory concentration required to inhibit the growth of $50 \%$ of organisms; MIC 90 , minimum inhibitory concentration required to inhibit the growth of $90 \%$ of organisms.

release peaked at 7 days, followed by gradual and sustained release. Further, the results showed that biodegradable nanofibrous membranes could effectively release high concentrations of vancomycin and ceftazidime, well above the MICs of the drugs, for more than 8 weeks (Figures 7 and 8).

\section{Creatinine and antibiotic levels in the blood}

The levels of serum creatinine and antibiotic concentrations were determined. Creatinine level was normal at each time point (Table 1), while the concentrations of vancomycin and ceftazidime in the blood remained higher than the MIC ( $2 \mu \mathrm{g} / \mathrm{mL}$ and $4 \mu \mathrm{g} / \mathrm{mL}$ for vancomycin and ceftazidime, respectively; Figures 7 and 8). While antibiotic concentration around the plate was high, the concentration in the blood was low. The results confirmed that the nanofibrous membranes

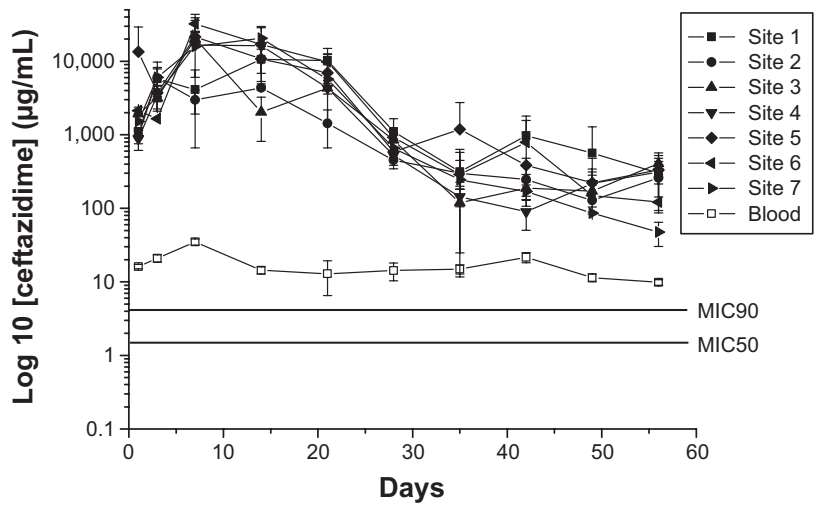

Figure 8 In vivo release behavior of ceftazidime.

Abbreviations: MIC50, minimum inhibitory concentration required to inhibit the growth of $50 \%$ of organisms; MIC 90 , minimum inhibitory concentration required to inhibit the growth of $90 \%$ of organisms.

loaded with vancomycin, which is associated with renal toxicity, could release high bactericidal concentrations of the drug in a sustained manner with a negligible effect on renal function.

\section{Histological examination}

Histological examination showed significant mononuclear cell infiltrates of lymphocytes, plasma cells, and eosinophils in the muscle tissue surrounding the plate at Day 1 postimplantation. The amount of polymorphonuclear leukocytes gradually decreased over 4 weeks (Figure 9).

\section{Discussion}

Infection, one of the most devastating complications resulting from an open fracture or an internal fixation, disturbs both patients and orthopedic surgeons. ${ }^{1-3}$ Although debridement

Table I Creatinine levels of experimental rabbits

\begin{tabular}{lllll}
\hline $\begin{array}{l}\text { Rabbit } \\
\text { numbers }\end{array}$ & $\begin{array}{l}\text { Days rabbits } \\
\text { sacrificed }\end{array}$ & $\begin{array}{l}\text { Creatinine levels before } \\
\text { the placement of the } \\
\text { PMMA spacer }(\mathbf{m g} / \mathbf{d L})\end{array}$ & $\begin{array}{l}\text { Creatinine levels before } \\
\text { the deployment of the } \\
\text { implants }(\mathbf{m g} / \mathbf{d L})\end{array}$ & $\begin{array}{l}\text { Creatinine levels on } \\
\text { sacrificed days after } \\
\text { the deployment of the } \\
\text { implants }(\mathbf{m g} / \mathbf{d L})\end{array}$ \\
\hline $1-3$ & 1 & $0.64-0.90$ & $0.65-1.01$ & $0.78-0.88$ \\
$4-6$ & 3 & $0.88-1.32$ & $0.76-1.43$ & $0.77-1.64$ \\
$7-9$ & 7 & $0.83-1.01$ & $0.86-1.11$ & $0.79-0.95$ \\
$10-12$ & 14 & $0.76-0.98$ & $0.77-1.12$ & $0.67-0.96$ \\
$13-15$ & 21 & $0.64-0.82$ & $0.92-1.44$ & $0.66-0.99$ \\
$16-18$ & 28 & $0.66-0.81$ & $0.78-0.93$ & $0.74-0.92$ \\
$19-21$ & 35 & $0.86-0.91$ & $0.92-1.01$ & $0.90-0.97$ \\
$22-24$ & 42 & $0.78-1.11$ & $0.86-1.07$ & $0.84-1.02$ \\
$25-27$ & 49 & $0.68-0.97$ & $0.98-1.03$ & $0.82-0.94$ \\
$28-30$ & 56 & $0.73-0.86$ & $0.88-0.98$ & $0.85-0.92$ \\
\hline
\end{tabular}

Note: Normal creatinine level: $0.5 \sim 2.6 \mathrm{mg} / \mathrm{dL}$.

Abbreviation: PMMA, polymethylmethacrylate. 

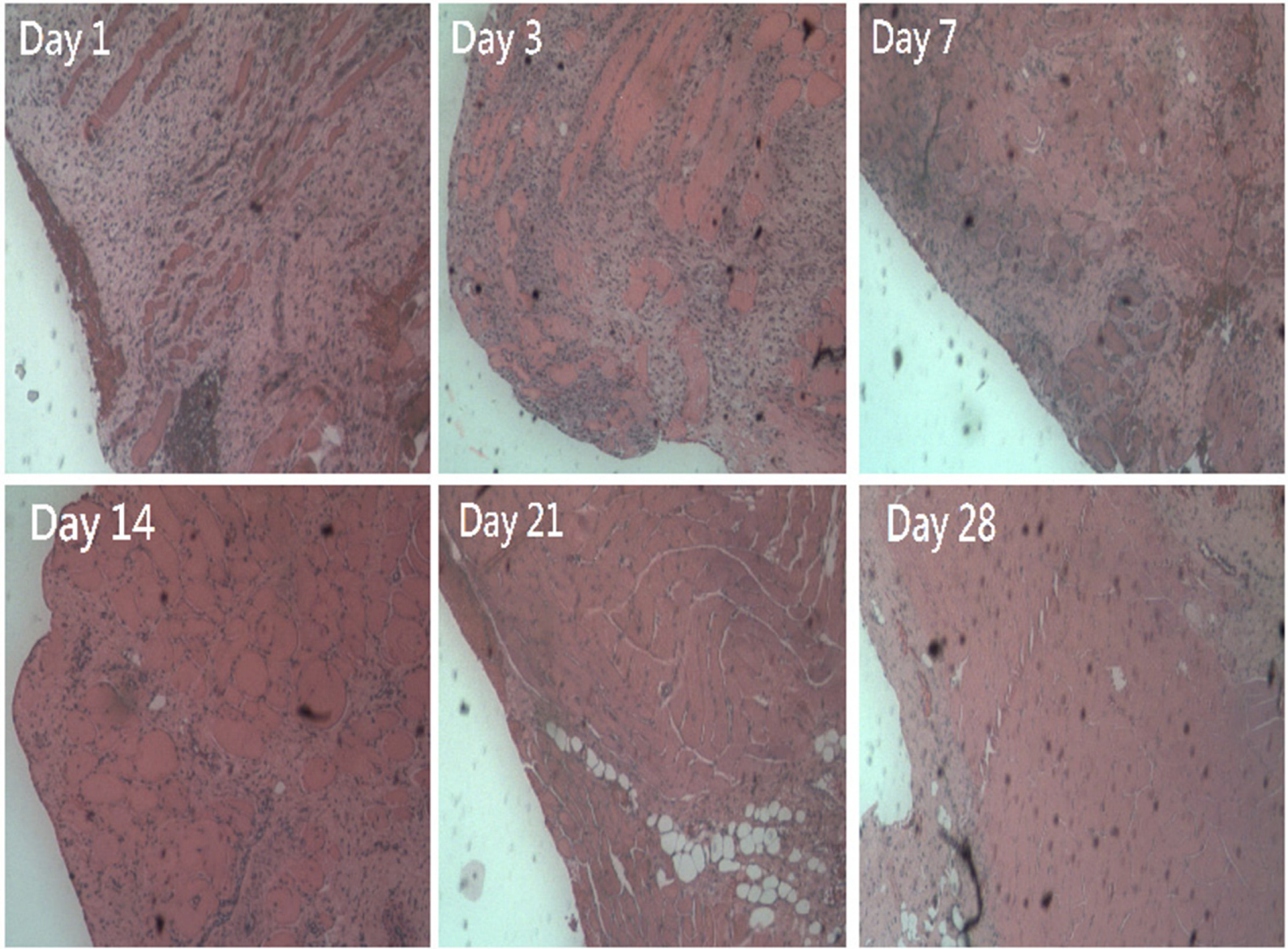

Figure 9 Histological examination of muscle tissue surrounding the plate at days I, 3, 7, 14, 21 , and 28 after surgery. Microscopic examination of hematoxylin-and-eosinstained specimens showed significant mononuclear cell infiltrates of lymphocytes, plasma cells, and eosinophils in the muscle tissue surrounding the plate at day I after surgery. The number of polymorphonuclear leukocytes gradually decreased over time to day 28 post-surgery.

Note: Original magnification $200 \times$.

and implant retaining have had some success in acute postoperative infection management following fracture internal fixation, ${ }^{15}$ the current standard surgical procedure for osteomyelitis treatment includes radical debridement, complete removal of the implant, and delivery of an effective antimicrobial agent at a sufficiently high concentration. The application of external skeletal fixation, such as the Hoffman device, in the interim stages has become a common approach. Furthermore, new operative techniques, such as the Ilizarov fixation, and innovative antibiotic delivery systems have been added to the treatment arsenal. The application of the Ilizarov fixation has been generated promising results. ${ }^{16} \mathrm{How}-$ ever, pin-site infection remains a possible complication. In addition, external fixation with a Hoffman fixator has other complications like bone shortening, malalignment, and decreased stability.

Several studies have shown promising results for the improvement of osteomyelitis treatment. Stewart et al developed a load-bearing biodegradable scaffold composed of polypropylene fumarate and tricalcium phosphate. ${ }^{8}$ The authors embedded gentamicin into a biodegradable scaffold and demonstrated its effectiveness in decreasing radiographically defined osteomyelitis in an infected open fracture. However, the fixed pin was not fully enveloped by the biodegradable antibiotic carrier and there was a possibility that bacteria may have grown on the antibiotic-free end of the pin. Stewart et al reported that vancomycin-derived plate surfaces inhibited implant colonization by $S$. aureus. ${ }^{6}$ Even though these plates showed promising inhibition of biofilm formation, bactericidal antibiotic concentration in the surrounding tissue was not demonstrated. In the study reported here, we developed biodegradable PLGA/antibiotic nanofibrous membrane-enveloped plates to provide sustained delivery of vancomycin and ceftazidime for infection treatment. The plate implantation procedure used is easy and familiar to every orthopedic surgeon. Furthermore, the biodegradable membrane can be easily applied to the plate and the screws (Figure 1). 
Various types of carrier materials have been used based on their ability to achieve sustained release of bactericidal concentrations of antibiotics. ${ }^{6-8}$ Nonbiodegradable PMMA antibiotic-impregnated beads are most commonly used in the treatment of osteomyelitis., ${ }^{417}$ However, when the drug is completely released, the PMMA beads act as a biomaterial surface to which bacteria adhere and grow, which might potentially cause the development of antibiotic resistance. ${ }^{18}$ These beads usually have to be removed with repeated surgery. There are many studies on biodegradable materials for drug-eluting systems. The base of the biodegradable drug-eluting system should be nonreactive and stable in body tissues. PLGA is promising biodegradable material that has been used for implantable and injectable controlled-release drug-delivery systems. ${ }^{8-10,17,19}$ In clinical application, the copolymer is nontoxic, induces minimal inflammatory response, and is biodegraded through hydrolysis without any accumulation in vital organs. ${ }^{10-12}$

$S$. aureus is the most common organism implicated in skeletal system infections. ${ }^{4,20}$ Approximately $20 \%$ of $S$. aureus isolates collected in Europe are reported to be methicillinresistant $S$. aureus, and, in US hospitals, the prevalence ranges from $33 \%$ to $55 \% .{ }^{21}$ Vancomycin, a glycopeptide antibiotic, remains the standard therapeutic agent for the treatment of Gram-positive pathogens, including resistant and nonresistant strains. ${ }^{20}$ Common Gram-negative bacteria involved in osteomyelitis include Pseudomonas aeruginosa, E. coli, and Klebsiella pneumoniae. ${ }^{14}$ Vancomycin and ceftazidime have been the antibiotics commonly formulated with bone cement for osteomyelitis treatment. ${ }^{13,14}$ In this study, drugeluting membranes were fabricated with PLGA, vancomycin, and ceftazidime. Based on the experimental results, the best composition for the PLGA/antibiotic nanofiber membranes is PLGA:vancomycin:ceftazidime $=240: 20: 20 \mathrm{mg}$ (Figures 4 and 5). The in vivo results showed that the biodegradable nanofibrous membranes released high concentrations of vancomycin and ceftazidime (well above the MIC) at Sites 1 to 7 and in the blood for 8 weeks (Figures 7 and 8). In addition, the drug concentrations in the blood also showed the potential "benign" side effect. Combined therapy involving local drug release by PMMA carriers and systemic drug delivery is commonly used in osteomyelitis treatment. The results of this study showed that the application of biodegradable drug-eluting membranes resulted in high local concentrations of antibiotics as well as systemic drug concentrations higher than the MIC. This might provide the advantage of saving the systemic delivery of antibiotics by intravascular injection. However, more studies are necessary to further investigate this benign side effect as well as the optimal drug doses for nanofibrous drug-eluting membranes.

Vancomycin is notorious for its renal toxicity. A high concentration of vancomycin in the blood might raise safety concerns regarding the use of high-dose antibioticeluting carriers. Springer et al reported that only one out of 36 patients undergoing a staged operation using vancomycingentamicin cement spacers had a transient rise in serum creatinine level. ${ }^{22}$ No other patients suffered systemic side effects associated with the use of the antibiotics. Acute renal failure after the implantation of a gentamicin-loaded Palacos ${ }^{\circledR}$ cement knee spacer has been reported in sporadic cases. ${ }^{23,24}$ In our study, creatinine level in the blood remained within the normal range, ${ }^{25}$ which indicated a negligible effect on renal function (Table 1). This further demonstrated the safety of the biodegradable drug-eluting membranes developed in this study.

In our study, we measured the antibiotic concentration at multiple sites and demonstrated that drug concentrations are high in the areas surrounding the plate and in the bone canal. We showed that the bioactivities of released vancomycin and ceftazidime remain high after the electrospinning process. This further confirmed that electrospinning could be an appropriate process for the preparation of drug-eluting membranes. Furthermore, the results demonstrated that high bactericidal concentrations could be achieved for a long period and indicated the areas around the plate were aseptic.

A major limitation of the present study is that a noninfected animal model was used. Whether the drug-eluting nanofiber-enveloped implants will perform differently in an infected area is not known; further evaluation of the drugeluting implants in an infection model is necessary to answer this question. In addition, the relevance of our findings to humans with bone infection remains unclear and needs to be further explored.

\section{Conclusion}

The biodegradable drug-eluting nanofibers release high bactericidal concentrations of vancomycin and ceftazidime for more than 32 days in vitro and 8 weeks in vivo. The preparation of drug-eluting membrane-enveloped plates is simple and the surgical technique is familiar to every orthopedic surgeon. Furthermore, the PLGA/antibiotic membranes released antibiotics at high concentration (well above MIC) locally and lower concentrations in the blood. The creatinine level remained within the normal range, suggesting that the vancomycin concentration in the blood did not affect the renal function. 


\section{Acknowledgments}

The authors would like to thank the National Science Council of Taiwan (Contract no. NSC-102-2314-B-182A030) and Chang Gung Memorial Hospital (Contract no. CMRPG391592) for financially supporting this research.

\section{Disclosure}

The authors report no conflicts of interest in this work.

\section{References}

1. Struijs PA, Poolman RW, Bhandari M. Infected nonunion of the long bones. J Orthop Trauma. 2007;21(7):507-511.

2. Crowley DJ, Kanakaris NK, Giannoudis PV. Debridement and wound closure of open fractures: the impact of the time factor on infection rates. Injury. 2007;38(8):879-889.

3. Trampuz A, Zimmerli W. Diagnosis and treatment of infections associated with fracture-fixation devices. Injury. 2006;37(Suppl 2):S59-S66.

4. Mader JT, Calhoun J, Cobos J. In vitro evaluation of antibiotic diffusion from antibiotic-impregnated biodegradable beads and polymethylmethacrylate beads. Antimicrob Agents Chemother. 1997; 41(2):415-418.

5. Ueng SW, Wei FC, Shih CH. Management of femoral diaphyseal infected nonunion with antibiotic beads local therapy, external skeletal fixation, and staged bone grafting. J Trauma. 1999;46(1):97-103.

6. Stewart S, Barr S, Engiles J, et al. Vancomycin-modified implant surface inhibits biofilm formation and supports bone-healing in an infected osteotomy model in sheep: a proof-of-concept study. J Bone Joint Surg Am. 2012;94(15):1406-1415.

7. Chen DW, Hsu YH, Liao JY, Liu SJ, Chen JK, Ueng SW. Sustainable release of vancomycin, gentamicin and lidocaine from novel electrospun sandwich-structured PLGA/collagen nanofibrous membranes. Int $J$ Pharm. 2012;430(1-2):335-341.

8. Stewart RL, Cox JT, Volgas D, et al. The use of a biodegradable, loadbearing scaffold as a carrier for antibiotics in an infected open fracture model. J Orthop Trauma. 2010;24(9):587-591.

9. Williams DF. Biodegradation of surgical polymers. J Mater Sci. 1982; 17:1233-1246.

10. Kumbar SG, Nukavarapu SP, James R, Nair LS, Laurencin CT. Electrospun poly(lactic acid-co-glycolic acid) scaffolds for skin tissue engineering. Biomaterials. 2008;29(30):4100-4107.

11. Ali SA, Doherty PJ, Williams DF. Mechanisms of polymer degradation in implantable devices. 2. Poly(DL-lactic acid). J Biomed Mater Res. 1993;27(11):1409-1418.
12. Kobayashi H, Shiraki K, Ikada Y. Toxicity test of biodegradable polymers by implantation in rabbit cornea. $J$ Biomed Mater Res. 1992;26(11):1463-1476.

13. Chan YS, Ueng SW, Wang CJ, Lee SS, Chao EK, Shin CH. Management of small infected tibial defects with antibiotic-impregnated autogenic cancellous bone grafting. J Trauma. 1998;45(4):758-764.

14. Chang Y, Tai CL, Hsieh PH, Ueng SW. Gentamicin in bone cement: a potentially more effective prophylactic measure of infectionin joint arthroplasty. Bone Joint Res. 2013;2(10):220-226.

15. Berkes M, Obremskey WT, Scannell B, Ellington JK, Hymes RA, Bosse M; Southeast Fracture Consortium. Maintenance of hardware after early postoperative infection following fracture internal fixation. J Bone Joint Surg Am. 2010;92(4):823-828.

16. Kocaoglu M, Eralp L, Rashid HU, Sen C, Bilsel K. Reconstruction of segmental bone defects due to chronic osteomyelitis with use of an external fixator and an intramedullary nail. J Bone Joint Surg Am. 2006;88(10):2137-2145.

17. Kontogiannopoulos KN, Assimopoulou AN, Tsivintzelis I, Panayiotou C, Papageorgiou VP. Electrospun fiber mats containing shikonin and derivatives with potential biomedical applications. Int $J$ Pharm. 2011;409(1-2):216-228.

18. Popham GJ, Mangino P, Seligson D, Henry SL. Antibiotic-impregnated beads. Part II: Factors in antibiotic selection. Orthop Rev. 1991; 20(4):331-337.

19. Meng ZX, Zheng W, Li L, Zheng YF. Fabrication, characterization and in vitro drug release behavior of electrospun PLGA/chitosan nanofibrous scaffold. Mater Chem Phys. 2011;125(3):606-611.

20. Chang Y, Chen WC, Hsieh PH, et al. In vitro activities of daptomycin-, vancomycin-, and teicoplanin-loaded polymethylmethacrylate against methicillin-susceptible, methicillin-resistant, and vancomycinintermediate strains of Staphylococcus aureus. Antimicrob Agents Chemother. 2011;55(12):5480-5484.

21. Appelbaum PC. MRSA - the tip of the iceberg. Clin Microbiol Infect. 2006;12(Suppl 2):3-10.

22. Springer BD, Lee GC, Osmon D, Haidukewych GJ, Hanssen AD, Jacofsky DJ. Systemic safety of high-dose antibiotic-loaded cement spacers after resection of an infected total knee arthroplasty. Clin Orthop Relat Res. 2004;(427):47-51.

23. van Raaij TM, Visser LE, Vulto AG, Verhaar JA. Acute renal failure after local gentamicin treatment in an infected total knee arthroplasty. $J$ Arthroplasty. 2002;17(7):948-950.

24. Curtis JM, Sternhagen V, Batts D. Acute renal failure after placement of tobramycin-impregnated bone cement in an infected total knee arthroplasty. Pharmacotherapy. 2005;25(6):876-880.

25. Melillo A. Rabbit clinical pathology. Journal of Exotic Pet Medicine. 2007;16(3):135-145.
International Journal of Nanomedicine

\section{Publish your work in this journal}

The International Journal of Nanomedicine is an international, peerreviewed journal focusing on the application of nanotechnology in diagnostics, therapeutics, and drug delivery systems throughout the biomedical field. This journal is indexed on PubMed Central, MedLine, CAS, SciSearch $®$, Current Contents $\AA /$ Clinical Medicine,

\section{Dovepress}

Journal Citation Reports/Science Edition, EMBase, Scopus and the Elsevier Bibliographic databases. The manuscript management system is completely online and includes a very quick and fair peer-review system, which is all easy to use. Visit http://www.dovepress.com/ testimonials.php to read real quotes from published authors. 